\title{
Identification of a Coding Sequence and Structure Modeling of a Glycine-Rich RNA-Binding Protein (CmGRP1) from Chelidonium majus $\mathrm{L}$.
}

\author{
Robert Nawrot • Lukasz Tomaszewski • \\ Anna Czerwoniec • Anna Goździcka-Józefiak \\ Published online: 13 September 2012 \\ (C) The Author(s) 2012. This article is published with open access at Springerlink.com
}

\begin{abstract}
The family of glycine-rich plant proteins (GRPs) is a large and complex group of proteins that share, as a common feature, the presence of glycine-rich domains arranged in (Gly)n-X repeats that are suggested to be involved in protein-protein interactions, RNA binding, and nucleolar targeting. These proteins are implicated in several independent physiological processes. Some are components of cell walls of many higher plants, while others are involved in molecular responses to environmental stress, and mediated by posttranscriptional regulatory mechanisms. The goals of this study are to identify the coding sequence of a novel glycine-rich RNA-binding protein from Chelidonium majus and to propose its structural model. DNA fragments obtained using degenerate PCR primers showed high sequence identities with glycine-rich RNA-binding protein coding sequences from different plant species. A 439-bp nucleotide sequence is identified coding for a novel polypeptide composed of 146 amino acids, designated as CmGRP1 (C. majus glycine-rich protein 1), with a calculated MW of 14,931 Da (NCBI GenBank accession no. HM173636). Using NCBI CDD and GeneSilico MetaServer, a single conserved domain, the RNA recognition motif (RRM), was
\end{abstract}

Accession numbers: the sequence reported in this article has been submitted to the GenBank database under accession number HM173636 (gi|380709245). The CmGRP1 protein_id is AEE42608.

R. Nawrot $(\varangle) \cdot$ Ł. Tomaszewski $\cdot$ A. Goździcka-Józefiak Department of Molecular Virology, Institute of Experimental Biology, Faculty of Biology, Adam Mickiewicz University in Poznań, Umultowska 89,

61-614 Poznań, Poland

e-mail: rnawrot@amu.edu.pl

\section{A. Czerwoniec}

Bioinformatics Laboratory, Institute of Molecular Biology and

Biotechnology, Faculty of Biology,

Adam Mickiewicz University in Poznań,

Umultowska 89,

61-614 Poznań, Poland detected in CmGRP1. The C-terminal region of CmGRP1 is a glycine-rich motif (GGGGxxGxGGGxxG), and it is predicted to be disordered. Based on a $1 \mathrm{fxl}$ crystal structure, a $3 \mathrm{D}$ model of CmGRP1 is proposed. CmGRP1 can be classified as a class IVa plant GRP, implicated to play a role in plant defense.

Keywords Glycine-rich protein · RNA-binding · Papaveraceae $\cdot$ Chelidonium majus Coding sequence . 3D model

\section{Introduction}

Glycine-rich plant proteins (GRPs) constitute a newly described group of proteins with diverse localizations and functions (Sachetto-Martins et al. 2000). They were first described as storage proteins, essentially being utilized as sources of amino acids for plants (Mousavi and Hotta 2005). GRPs are associated with dormancy, and are similar to proteins that are stimulated by abiotic stresses. Some are components of cell walls of higher plants and accumulate in vascular tissues as part of defense mechanisms against pathogens and wounding, and also play an important role in post-transcriptional regulation of gene expression (Ringli et al. 2001; Mousavi and Hotta 2005). More than 150 different GRP genes have been identified following transcriptome or whole genome analysis of sugarcane, eucalyptus, rice and Arabidopsis (Mangeon et al. 2009, 2010). Their expression is developmentally regulated, and also induced by various chemical, physical and biological factors (Mousavi and Hotta 2005), such as cold, water stress, drought, wounding, and in response to bacterial, viral and fungal infection (Cornels et al. 2000; Park et al. 2000; Ringli et al. 2001; Wang et al. 2011). GRPs are characterized by the presence of a glycine-rich domain arranged in (Gly)n-X repeats, which are highly flexible and may act in proteinprotein interactions (Sachetto-Martins et al. 2000). The 
presence of additional motifs, as well as the nature of glycine repeats, groups them into four major classes (Bocca et al. 2005). GRPs from class I contain a signal peptide followed by a glycine-rich region with GGX repeats. Most of these proteins are cell wall localized. Class II GRPs may or may not have a signal peptide and contain a glycine-rich region followed by a cysteine-rich region at their C-terminus. Class III GRPs contain proteins with lower glycine content, depicting a great diversity of structures. Class IV GRPs comprise RNAbinding domain proteins. The RNA-binding domain has one RNA recognition motif (RRM) at the amino (N) terminal, followed by a carboxy (C) terminal region composed of up to $70 \%$ glycine residues, interrupted mostly by arginine or aromatic amino acid residues, and contains arginine-glycinerich (RGG) motifs. RGG domains participate in protein-protein interaction and nucleolar targeting. These GRPs may also contain a cold-shock domain (CSD) and zinc fingers (CCHC) (Fusaro et al. 2001; Mangeon et al. 2010). Thus, class IV GRPs can be classified into four different sub-classes based on diversity of domain arrangements. Proteins that contain one RRM motif besides the glycine-rich domain belong to subclass IVa; subclass IVb has one RRM and a CCHC zinc-finger; subclass IVc has a CSD and two or more zinc-fingers; and subclass IVd has two RRMs (Mangeon et al. 2010).

RRMs are found in a variety of RNA-binding proteins, including various heterogeneous nuclear ribonucleoproteins (hnRNPs), proteins implicated in the regulation of alternative splicing, and protein components of small nuclear ribonucleoproteins (snRNPs). The motif also appears in a few singlestranded DNA binding proteins (Albà and Pagès 1998).

Chelidonium majus L. (Greater Celandine) belongs to the Papaveraceae family and is a rich source of various biologically active substances. All of them occur in the milky sap - a milkylike orange fluid, similar to latex, isolated from the opium poppy (Papaver somniferum) (Decker et al. 2000). Recent findings using two-dimensional electrophoresis (2-DE) and tandem mass spectrometry analysis (LC-MS/MS) have demonstrated that the milky sap of this plant contains about 20 defence-related proteins (Nawrot et al. 2007). One of them was identified as a GRP that binds to nucleic acids, similar to AtGRP2B (glycine-rich protein 2B; DNA binding/nucleic acid binding) with MW of $19.4 \mathrm{kDa}$ (Nawrot et al. 2007).

The goals of this study were to identify the coding sequence of a novel glycine-rich RNA-binding protein from Chelidonium majus and propose a model for its structure.

\section{Materials and Methods}

DNA Isolation and PCR Amplification

Genomic DNA was isolated from 14-day-old C. majus seedlings using a DNeasy Plant Mini Kit (Qiagen, Hilden,
Germany). To obtain the GRP gene sequence, a polymerase chain reaction (PCR) was carried out using degenerate primers GRP1 (5'-TGYTTYGTNGGNGGNCTNG-3') and GRP4 (5'-CCNCCNCCRTANCC-3') designed on the basis of highly conserved regions of GRP (Nomata et al. 2004). PCR was performed in a TGradient thermocycler (Biometra, Göttingen, Germany). Initial DNA denaturation was performed at $95{ }^{\circ} \mathrm{C}$ for $5 \mathrm{~min}$, then step 2 denaturation at $95{ }^{\circ} \mathrm{C}$ for $15 \mathrm{~s}$, followed by primer annealing at $53.4{ }^{\circ} \mathrm{C}$ for $30 \mathrm{~s}$ and elongation at $72{ }^{\circ} \mathrm{C}$ for $120 \mathrm{~s}$. This cycle of denaturation, annealing and elongation was repeated 35 times and followed by final elongation at $72^{\circ} \mathrm{C}$ for $15 \mathrm{~min}$.

\section{Ligation, Transformation and Sequencing}

Purified PCR products were ligated into the vector pGEM-T Easy (Promega, Madison, WI). Competent Escherichia coli DH5 $\alpha$ cells were transformed with ligation products. Plasmids with inserts were extracted with a QIAprep Plasmid Kit (Qiagen) from white transformed colonies and used as templates for PCR amplification using standard M13 primers (Promega). Inserts were sequenced with an automated 3130× Genetic Analyzer (Applied Biosystems, Foster City, CA) at the Faculty of Biology, Adam Mickiewicz University in Poznań, Poland. DNA sequences were analyzed using VectorNTI (Invitrogen, Carlsbad, CA), NCBI VecScreen, BLASTn and BLASTx tools (http://www.ncbi.nlm.nih.gov).

\section{Protein Structure Prediction and Modeling}

Tertiary structure prediction and fold-recognition was carried out via the GeneSilico MetaServer gateway (Kurowski and Bujnicki 2003). The top-scoring fold-recognition alignments to the structures of the selected template were used as a starting point for homology modeling using the "Frankenstein's Monster" approach (Kosinski et al. 2003, 2005), comprising cycles of model building, evaluation, realignment in poorly scored regions and merging of the best scoring fragments.

For model evaluation, two model quality assessment programs (MQAPs) were used: MetaMQAP (Pawlowski et al. 2008) and PROQ (Wallner and Elofsson 2003). MQAP scores only predict the deviation of a model from the real structure (the real deviation can be calculated only by comparison with the real structures, which of course are not available). Thus, scores reported in this study that indicate, e.g., 'very good models', must be interpreted as estimations or predictions that our models are 'very good', and not as ultimate validation of the model quality. However, it should be mentioned that both PROQ and MetaMQAP performed quite well in independent benchmarks and can be regarded as robust predictors. 


\section{Results}

The Search for C. majus GRP Coding Sequence

In order to obtain the coding sequence of a putative $C$. majus glycine-rich RNA-binding protein, amplification products were obtained after degenerate PCR using a GRP1f/GRP4r primer pair with a gradient of primer annealing temperature. Primers were designed based on a highly conserved RRM domain of known GRP proteins (Nomata et al. 2004). Due to the low quantity, PCR products were recovered from the agarose gel and served as templates for re-PCR using the same set of primers. Specific re-PCR products were obtained in reactions with primers annealing at $53.4{ }^{\circ} \mathrm{C}$. These were ligated into pGEM-T Easy vector (Promega) and cloned in E. coli DH5 $\alpha$ cells. Plasmids were extracted from white transformed colonies and used as templates for PCR amplification using standard M13 primers (Promega). The presence of inserts was confirmed for plasmids extracted from six separate colonies. The inserts were sequenced and analysed using bioinformatic tools. Contaminating vector sequences were eliminated using NCBI VecScreen and the resulting $300 \mathrm{bp}$ sequence was further analysed using BLASTn and BLASTp tools (http://www.ncbi.nlm.nih.gov). The full-length GRP gene sequence was obtained by a series of PCR reactions with the use of primers complementary to known fragments of the sequence and to regions upstream and downstream based on the homologous sequences from other plant species. High sequence identity to glycinerich RNA-binding protein coding sequences from different plants (e.g., Glycine max, Arabidopisis thaliana) was found (about $73 \%$ maximum identity, 154 total score and E-value of 2e-34). The 439 bp nucleotide sequence coding for the novel GRP (named CmGRP1: Chelidonium majus glycine-rich protein 1) composed of 146 amino acids has been submitted to the GenBank database under accession number HM173636 (gi|380709245, protein_id AEE42608). The calculated molecular weight of the novel polypeptide was 14,931 Da.

Multiple sequence alignment showed the high sequence similarity of the CmGRP1 translated amino acid sequence to different glycine-rich RNA-binding proteins from different plant species (Fig. 1).

\section{CmGRP1 Domain Architecture}

The presence of conserved domains in the CmGRP1 protein was predicted from information gathered from NCBI CDD (Marchler-Bauer et al. 2009) and GeneSilico MetaServer (Kurowski and Bujnicki 2003; Piszczek et al. 2012). One conserved domain, the RNA recognition motif (RRM, RBD, or RNP domain), was detected. RRM domains are found in a variety of RNA-binding proteins. The RRM domain
Fig. 1 Amino acid sequence alignment of representatives of glycinerich binding proteins (GRPs). CmGRPI Translated amino acid sequence of the Chelidonium majus GRP gene obtained in this study. Alignments of amino acid sequences of various known GRPs were created by the ClustalX program. The following sequences were obtained from the GenBank database: glycine-rich RNA binding protein (Zea mays); putative glycine rich RNA binding protein (Solanum tuberosum); putative glycine-rich RNA binding protein 1 (Catharanthus roseus); RNA-binding glycine-rich protein 1-c (Nicotiana sylvestris); glycine-rich RNA-binding protein (Glycine max); glycine-rich RNA binding protein 7 (Arabidopsis thaliana); glycine-rich RNAbinding protein GRP1A (Sinapis alba); hypothetical protein OsI_12955 (Oryza sativa Indica Group); single-stranded nucleic acid binding protein (Triticum aestivum); 1FXL chain A-crystal structure of HUD and AU-rich element of the C-FOS RNA (Homo sapiens). NCBI gene identification numbers are presented in the figure with abbreviated genus and species names. Secondary structure prediction for CmGRP1 protein is shown as arrows and tubes (strands and helices, respectively). The blocks below the sequences indicate conserved motifs (RRM and glycine-rich domain). The positions of conserved residues are colored by two grade shading with black (identical residues) and grey (similar residues). The shading of amino acids in columns is for $60 \%$ threshold values of amino acids similarity

consists of four strands and two helices arranged in an alpha/beta sandwich, with a third helix present during RNA binding in some cases (SCOP: Fold-Ferredoxin-like). The C-terminal region of the CmGRP1 protein is a glycinerich motif (GGGGxxGxGGGxxG) and is predicted to be disordered. Based on the classification of plant GRPs, CmGRP1 can be classified into the IVa class (Mangeon et al. 2010).

Sequence similarities between selected homologue sequences are shown in Fig. 1. On the basis of this alignment, a preliminary tree was calculated using the neighborjoining approach implemented in MEGA (Kumar et al. 2004) (Fig. 2). CmGRP1 has a high sequence identity (80\%) and similarity (87\%) to the glycine-rich RNAbinding protein from Glycine max, and AtGRP7 from $A$. thaliana (76\% and $87 \%$, respectively) (Fig. 2).

\section{CmGRP1 Protein Structure Prediction and Modeling}

Based on the fold-recognition (FR) alignment proposed by GeneSilico MetaServer, template structures were selected [PDB: $1 \times 5 \mathrm{~s}$ chain A-solution structure of RRM domain in A18 hnRNP (Homo sapiens), mgenthreaderscore 3e-05, PDB: $1 \mathrm{fxl}$ chain A-crystal structure of HUD and an AU-rich element of the C-FOS RNA (Homo sapiens), pdbblast-score 6e-21, blastp-score 3e08 and 1rk8 chain A-structure of the cytosolic protein PYM bound to the Mago-Y14 core of the exon junction complex (Drosophila melanogaster), hhsearch-score 99.94]. The sequence identity in the aligned regions of CmGRP1 and $1 \mathrm{fxl}, 1 \times 5 \mathrm{~s}$ and $1 \mathrm{rk} 8$ is $36 \%, 45 \%$ and $18 \%$, respectively. All templates are classified as alpha and beta proteins with a ferredoxin-like fold belonging 
CmGRP 1

198282085 | ZEAMAY |

799015 | SOLTUB |

6911142 | CATROS |

469072 | NICSYL |

5726567 | GLYMAX |

30681492 | ARATHA |

1346180 | SINALB |

218193471 | ORYSAT

974605 | TRIAES |

1FXL

CmGRP1 SS

CDD

\section{CmGRP1}

198282085 | ZEAMAY

799015 | SOLTUB |

6911142 | CATROS |

469072 | NICSYL |

5726567 | GLYMAX |

30681492 |ARATHA |

1346180 | SINALB |

218193471 |ORYSAT

974605 | TRIAES |

$1 \mathrm{FXL}$

CmGRP1 SS

CDD

\section{CmGRP1}

198282085 | ZEAMAY 799015 | SOLTUB | 6911142 | CATROS | 469072 | NICSYL | 5726567 | GLYMAX | 30681492 |ARATHA | 1346180 | SINALB | 218193471 |ORYSAT 974605 | TRIAES |

$1 \mathrm{FXL}$

CmGRP1_SS

CDD
10

20

30

40

50

60
MASADVEYRCCFVGGLAWATDDQSLEKAFSSYRGIVESKIINDRETCWSRGFGFVTFANE MAASDVEYR-CFVGGLAWATDDHSLNNAF STYGEVLESKI ILDRETQRSRGF GFVTFSTE - -MADVEYR-CFVGGLAWATTDQTLSDAF SQYAEVVESK I INDRETGRSRGFGFVTFKDE MASADVEFR-CFVGGLAWATTDQSLSEAFSOYGEVLESKI INDRETGRSRGFGFVTFGDE - -MAEVEYR-CFVGGLAWATTDRTLGEAF SQYGEVLESKI INDRETGRSRGFGFVTFGDE MASADVEYR-CFVGGLAWATDDHALERAF SOYGEIVETKI INDRETGRSRGFGFVTFASE MAS SDVEYR-CFVGGLAWATDDRALETAFAQYGDVIDSKI INDRETGRSRGF GFVTFKDE MASPDVEYR-CFVGGLAWATDDRALETAFSQYGEVLDSKI INDRETGRSRGF GFVTFKDE MAAPDVEYR-CFVGGLAWATDDRSLEAAFSTYGEIIDSKI INDRETGRSRGF GFVTFSSE - -MAETEYR-CFVGGLAWATDDNNLQQAF SQYGEILDAKI INDRETGRSRGFGFVTFGSE - - - - SKTN - LIVNYLPQNMTQEEFRSLFGSIGEIESCKLVRDKITGQSLGYGFVNYIDP

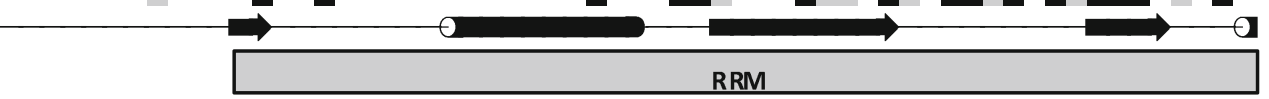

20

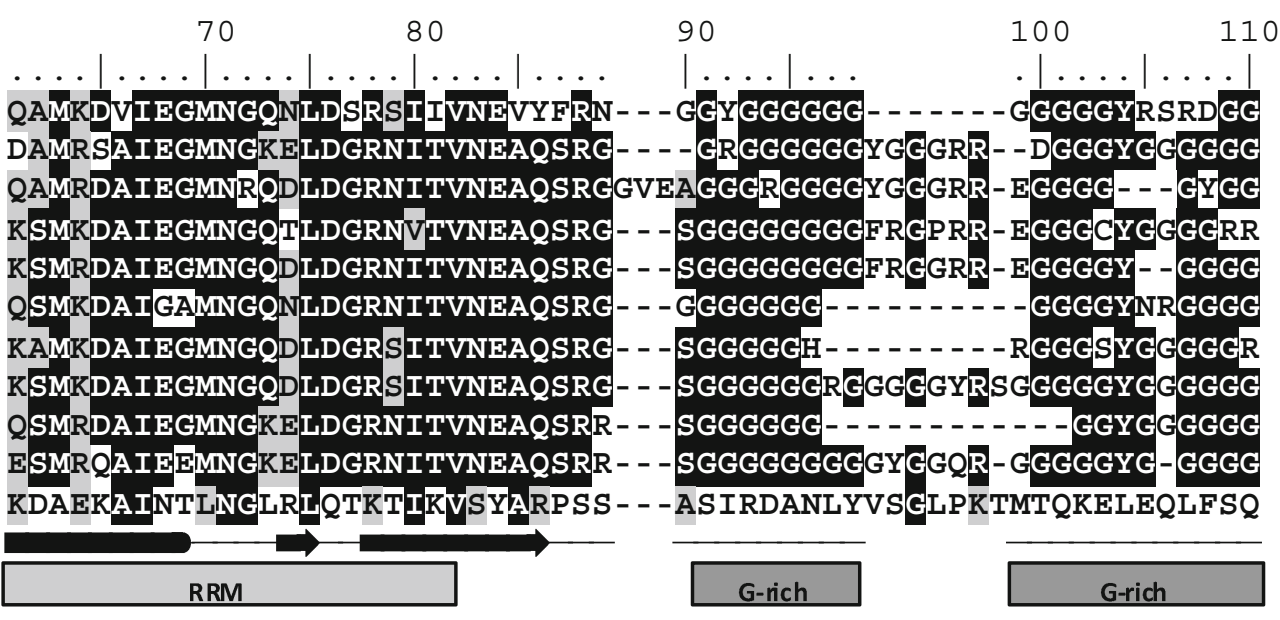

to RNA-binding domain superfamily (SCOP). This allows us to employ a homology modeling approach for constructing 3D protein structures.

The modeled structure of the N-terminal fragment (1-89 aa, RRM domain) of protein CmGRP1 was evaluated as a 'fairly good model' by the PROQ method for model quality prediction [predicted LGscore: 2.378 (LGscore is - log of a $P$-value)]. The MetaMQAP method predicted that the overall GDT_TS score (global distance test total score-a measure of similarity between two protein structures with 
identical amino acid sequences, but different tertiary structures) of the model with respect to the native structure is 75.3 and RMSD 1.8, indicating that this model can be used to deduce functional inferences. Figure 3 (panel B) illustrates the predicted quality of different regions of the protein structure.

For CmGRP1, sequence predictions of protein-RNA interaction were conducted. Methods implemented on GeneSilico MetaServer suggested the following amino acids: 46-50, 88-140 (glycine-rich and disordered region). To confirm the predicted and present structures in the template, the Haddock protein-RNA interaction method was used (Haddock web server; de Vries et al. 2010). A RNA molecule from the $1 \mathrm{fxl}$ crystal structure was docked flexibly to the CmGRP1 model (Wang and Tanaka Hall 2001). The best model from a highly represented cluster (HADDOCK score: $-63.2+/-6.4$, RMSD from the overall lowest-energy structure: $1.6+/-1.0$, Van der Waals energy: $-32.9+/-6.5$, electrostatic energy: $-128.4+/-9.8$, desolvation energy: $-6.4+/-4.2$, restraint violation energy: $18.5+/-16.61$, buried surface area: $997.6+/-110.6$ ) was selected (Fig. 3). Detailed interactions for protein region $43-50$ as well as polar contacts between side chains (E45, T46, R50) and RNA are shown in Fig. 3c.

\section{Discussion}

Glycine-rich RNA-binding proteins (GRRBPs), which are found primarily in plants as well as in some primate species, are classified on the basis of the presence of a C-terminal glycine enriched region and have extremely diverse and independent functions. Many members of this protein family have a function in stress responses (Kim et al. 2010; Mangeon et al. 2010; Wang et al. 2011). The calculated MW of the novel polypeptide identified here was ca. $15 \mathrm{kDa}$ $(14,931 \mathrm{Da})$. Based on its domain architecture (Fig. 1), CmGRP1 was suggested to belong to class IVa of plant GRPs, which are implicated to play a role in plant defense (Mangeon et al. 2010). CmGRP1 has a high sequence identity (76\%) and similarity (87\%) to AtGRP7 from A. thaliana (Figs. 1, 2). GRRBPs AtGRP7 and AtGRP8 were upregulated rapidly in response to peroxideinduced oxidative stress (Schmidt et al. 2009). Another example of the involvement of GRPs in plant defense comes from studies on the interaction between Arabidopsis thaliana and Pseudomonas syringae. Biochemical analysis indicates that two Class IVa GRPs, AtGRP7 and AtGRP8, are ribosylated in vitro by a type III effector protein during infection by $P$. syringae, resulting in quelled plant immunity. This type III effector is a mono-ADP-ribosyltransferase (HopU1-His) that targets
RNA-binding proteins presenting RRM motifs as substrates (Fu et al. 2007).

Using GeneSilico MetaServer, a 3D model of CmGRP1 was proposed on the basis of the $1 \mathrm{fxl}$ chain A crystal structure (Fig. 3). HuD, a 167AA protein, binds to adenosine-uridine (AU)-rich elements (AREs) in the $3^{\prime}$ untranslated regions of many short-lived mRNAs, thereby stabilizing them (Wang and Tanaka Hall 2001). Hu proteins contain three highly conserved RRM domains. The first two RRMs are in tandem and are necessary and sufficient for binding to ARE (Wang and Tanaka Hall 2001). For CmGRP1, sequence predictions for protein-RNA interaction were conducted based on the $\mathrm{HuD}$ protein in complex with an 11-nucleotide fragment of a class I ARE (the c-fos ARE). An RNA molecule from the $1 \mathrm{fxl}$ crystal structure was docked flexibly to the CmGRP1 model confirming the quality of the model (Fig. 3; Wang and Tanaka Hall 2001).

CmGRP1 contains an RRM, found in a variety of canonical RNA-binding proteins. These include hnRNPs implicated in the regulation of alternative splicing, and protein components of snRNPs - central players in mRNA splicing. The motif also appears in a few single-stranded DNA-binding proteins. The RRM structure consists of four strands and two helices arranged in an alpha/beta sandwich, and a third helix in some cases present during RNA-binding (Albà and Pagès 1998). RRM has two highly conserved ribonucleoprotein (RNP) motif sequences. RNP-1 consists of eight amino acid residues and RNP-2 contains six amino acid residues (Nomata et al. 2004). Amino acid residues in RNP-1, RNP-2 and glycine-rich domains are involved in RNA binding (Nomata et al. 2004). RNA-binding proteins are ubiquitous cellular proteins that regulate gene expression mainly at the post-transcriptional level, which involves pre-mRNA splicing, nucleocytoplasmic mRNA transport, mRNA stability and decay, and translation (Kim et al. 2010). It is possible that GRRBPs, as members of RNA-binding proteins (RBPs), act as regulators of RNA processing and/or stability for mRNAs that are highly expressed during stress conditions (Albà and Pagès 1998; Zhang et al. 2011). This, in turn, would lead to the accumulation of stress-related proteins and secondary metabolites with a protective function. RNA is structurally very flexible and proteins, named RNA chaperones, can assist RNAs in reaching their functionally active states in vivo, e.g., by facilitation or prevention of RNA-RNA interactions (Lorković 2009).

In this study, we identified a 439-bp nucleotide sequence coding for a novel polypeptide, named CmGRP1, composed of 146 amino acids from C. majus. The protein could be classified into class IVa of plant GRPs, which have a role in plant defense. A 3D model of CmGRP1 based on the $1 \mathrm{fxl}$ 
Fig. 2 Phylogenetic tree of selected homologs of CmGRP1. Selected sequences are indicated by their abbreviated genus and species names (e.g., ARATHA for Arabidopsis thaliana) and the NCBI gene identification (GI) number. See Fig. 1 legend for all genus and species names. Bootstrap support for different nodes is shown

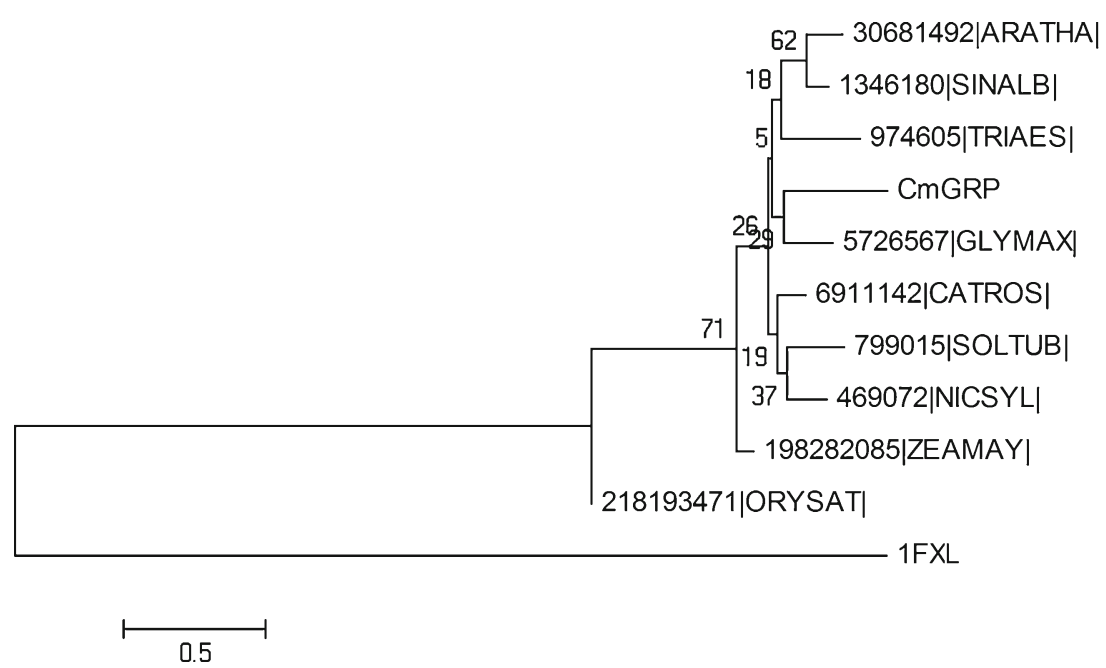

chain A crystal structure was proposed and showed a good correlation of CmGRP1 with the RNA molecule. These results could form the basis for further studies on the

possible correlation of the nucleolytic activity of $C$. majus milky sap with presence of nucleic acid-binding proteins and their role in plant defense.

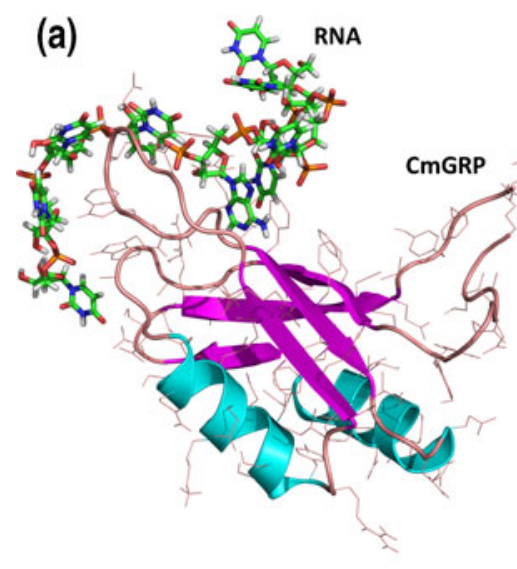

(d)

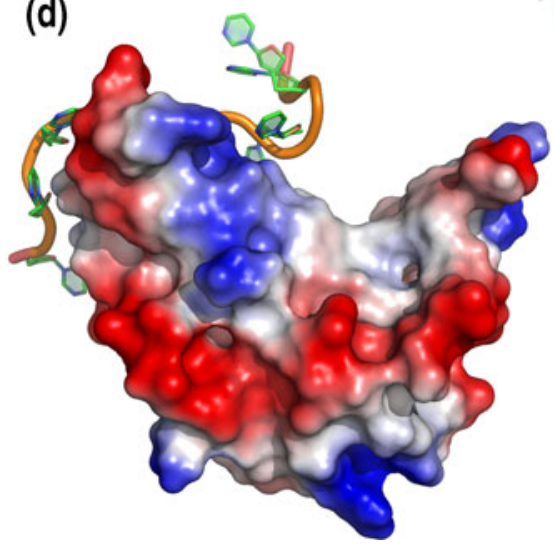

Fig. 3 Model of the CmGRP1 protein in a complex with an RNA molecule. All representations of a given protein are shown to the same scale, the RNA molecule is from a $1 \mathrm{fxl}$ crystal structure. a Model in a ribbon representation with side chains. $\mathbf{b}$ Model in a ribbon representation, colored according to the predicted local deviation from the real structure (i.e., the predicted error of the model), as calculated by MetaMQAP. Blue low predicted deviation of $\mathrm{C} \alpha$ atoms down to $0 \AA$,

(c)

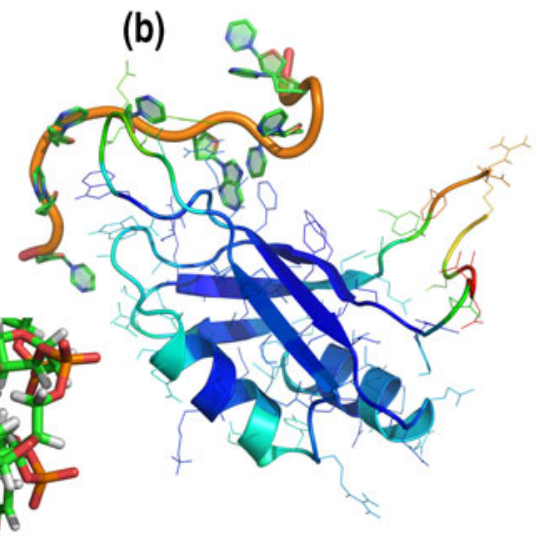

(e)
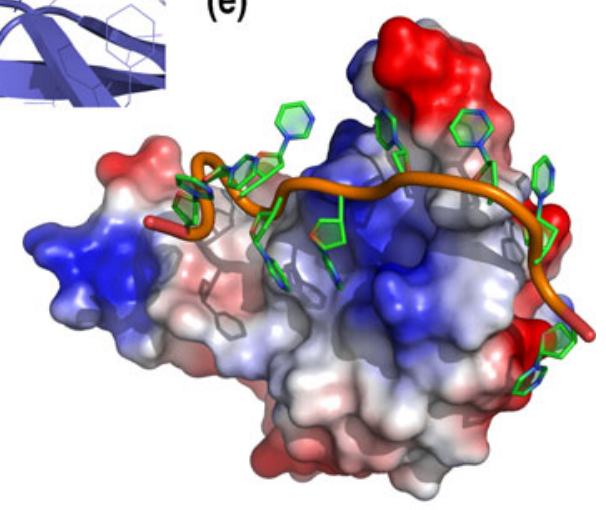

red unreliable regions with deviation $>5 \AA$, green-to-orange intermediate values. c Fragment or protein and RNA molecule with polar contacts. d, e Model in two different orientations in the surface representation, colored according to the distribution of the electrostatic surface potential calculated with ABPS (PyMol). Blue positively charged regions, red negatively charged regions 
Acknowledgment This work was supported by Polish Ministry of Science and Higher Education grant no. N N303 087234, 2008-2011.

Open Access This article is distributed under the terms of the Creative Commons Attribution License which permits any use, distribution, and reproduction in any medium, provided the original author(s) and the source are credited.

\section{References}

Albà MM, Pagès M (1998) Plant proteins containing the RNArecognition motif. Trends Plant Sci 3:15-21

Bocca SN, Magioli C, Mangeon A, Junqueira RM, Cardeal V, Margis R, Sachetto-Martins G (2005) Survey of glycine-rich proteins (GRPs) in the Eucalyptus expressed sequence tag database (ForEST). Genet Mol Biol 28:608-624

Cornels H, Ichinose Y, Barz W (2000) Characterization of cDNAs encoding two glycine-rich proteins in chickpea (Cicer arietinum L.): accumulation in response to fungal infection and other stress factors. Plant Sci 154:83-88

de Vries SJ, van Dijk M, Bonvin AMJJ (2010) The HADDOCK web server for data-driven biomolecular docking. Nat Protocol 5:883-897

Decker G, Wanner G, Zenk MH, Lottspeich F (2000) Characterization of proteins in latex of the opium poppy (Papaver somniferum) using two-dimensional gel electrophoresis and microsequencing. Electrophoresis 21:3500-3516

Fu ZQ, Guo M, Jeong BR, Tian F, Elthon TE, Cerny RL, Staiger D, Alfano JR (2007) A type III effector ADP-ribosylates RNAbinding proteins and quells plant immunity. Nature 447:284-288

Fusaro A, Mangeon A, Magrani Junqueira R, Benicio Rocha CA, Cardoso Coutinho T, Margis R, Sachetto-Martins G (2001) Classification, expression pattern and comparative analysis of sugarcane expressed sequences tags (ESTs) encoding glycine-rich proteins (GRPs). Genet Mol Biol 24:263-273

Kim JY, Kim WY, Kwak KJ, Oh SH, Han YS, Kang H (2010) Glycinerich RNA-binding proteins are functionally conserved in Arabidopsis thaliana and Oryza sativa during cold adaptation process. J Exp Bot 61:2317-2325

Kosinski J, Cymerman IA, Feder M, Kurowski MA, Sasin JM, Bujnicki JM (2003) A "FRankenstein's monster" approach to comparative modeling: merging the finest fragments of Fold-Recognition models and iterative model refinement aided by $3 \mathrm{D}$ structure evaluation. Proteins 53(Suppl 6):369-379

Kosinski J, Gajda MJ, Cymerman IA, Kurowski MA, Pawlowski M, Boniecki M, Obarska A, Papaj G, Sroczynska-Obuchowicz P, Tkaczuk KL et al (2005) FRankenstein becomes a cyborg: the automatic recombination and realignment of fold recognition models in CASP6. Proteins 61(Suppl 7):106-113

Kumar S, Tamura K, Nei M (2004) MEGA3: integrated software for molecular evolutionary genetics analysis and sequence alignment. Brief Bioinform 5:150-163

Kurowski MA, Bujnicki JM (2003) GeneSilico protein structure prediction meta-server. Nucleic Acids Res 31:3305-3307
Lorković ZJ (2009) Role of plant RNA-binding proteins in development, stress response and genome organization. Trends Plant Sci 14:229-236

Mangeon A, Magioli C, Menezes-Salgueiro AD, Cardeal V, de Oliveira C, Galvão VC, Margis R, Engler G, Sachetto-Martins G (2009) AtGRP5, a vacuole-located glycine-rich protein involved in cell elongation. Planta 230:253-265

Mangeon A, Junqueira RM, Sachetto-Martins G (2010) Functional diversity of the plant glycine-rich proteins superfamily. Plant Signal Behav 5:99-104

Marchler-Bauer A, Anderson JB, Chitsaz F, Derbyshire MK, DeWeeseScott C, Fong JH, Geer LY, Geer RC, Gonzales NR, Gwadz M et al (2009) CDD: specific functional annotation with the conserved domain database. Nucleic Acids Res 37(Database issue):D205-D210

Mousavi A, Hotta Y (2005) Glycine-rich proteins: a class of novel proteins. Appl Biochem Biotechnol 120:169-174

Nawrot R, Kalinowski A, Gozdzicka-Jozefiak A (2007) Proteomic analysis of Chelidonium majus milky sap using two-dimensional gel electrophoresis and tandem mass spectrometry. Phytochemistry 68:1612-1622

Nomata T, Kabeya Y, Sato N (2004) Cloning and characterization of glycine-rich RNA-binding protein cDNAs in the moss Physcomitrella patens. Plant Cell Physiol 45:48-56

Park CJ, Park CB, Hong SS, Lee HS, Lee SY, Kim SC (2000) Characterization and cDNA cloning of two glycine- and histidine-rich antimicrobial peptides from the roots of shepherd's purse, Capsella bursa-pastoris. Plant Mol Biol 44:187-197

Pawlowski M, Gajda MJ, Matlak R, Bujnicki JM (2008) MetaMQAP: a meta-server for the quality assessment of protein models. BMC Bioinformatics 9:403

Piszczek E, Dudkiewicz M, Mielecki M (2012) Biochemical and bioinformatic characterization of type II metacaspase protein (TaeMCAII) from wheat. Plant Mol Biol Rep. doi:10.1007/ s11105-012-0450-6

Ringli C, Keller B, Ryser U (2001) Glycine-rich proteins as structural components of plant cell walls. Cell Mol Life Sci 58:1430-1441

Sachetto-Martins G, Franco LO, de Oliveira DE (2000) Plant glycinerich proteins: a family or just proteins with a common motif? Biochim Biophys Acta 1492:1-14

Schmidt F, Marnef A, Cheung MK, Wilson I, Hancock J, Staiger D, Ladomery M (2009) A proteomic analysis of oligo(dT)-bound mRNP containing oxidative stress-induced Arabidopsis thaliana RNAbinding proteins ATGRP7 and ATGRP8. Mol Biol Rep 37:839-845

Wallner B, Elofsson A (2003) Can correct protein models be identified? Protein Sci 12:1073-1086

Wang X, Tanaka Hall TM (2001) Structural basis for recognition of AUrich element RNA by the HuD protein. Nat Struct Biol 8:141-145

Wang S, Liang D, Shi S, Ma F, Shu H, Wang R (2011) Isolation and characterization of a novel drought responsive gene encoding a glycine-rich RNA-binding protein in Malus prunifolia (Willd.) Borkh. Plant Mol Biol Rep 29:125-134

Zhang X, Zhen JB, Li ZH, Kang DM, Yang YM, Kong J, Hua JP (2011) Expression profile of early responsive genes under salt stress in upland cotton (Gossypium hirsutum L.). Plant Mol Biol Rep 29:626-637 